\title{
L Score as a Novel Anthropometric Measure for Obesity Screening in Adult Individuals: An Exploratory Study
}

\author{
Devi Prasad Mohapatra ${ }^{1}$, Jaya Prakash Sahoo², Madhusmita Mohanty Mohapatra ${ }^{3 *}$, Sitanshu Shekhar Kar ${ }^{4}$, \\ Sridharan Kalyani ${ }^{2}$, Ayan Roy ${ }^{2}$
}

${ }^{1}$ Department of Plastic Surgery, JIPMER, Puducherry, 605006, India; ${ }^{2}$ Department of Endocrinology, JIPMER, Puducherry, 605006, India; ${ }^{3}$ Department of Pulmonary Medicine, JIPMER, Puducherry, 605006, India; 4Department of Community Medicine, JIPMER, Puducherry

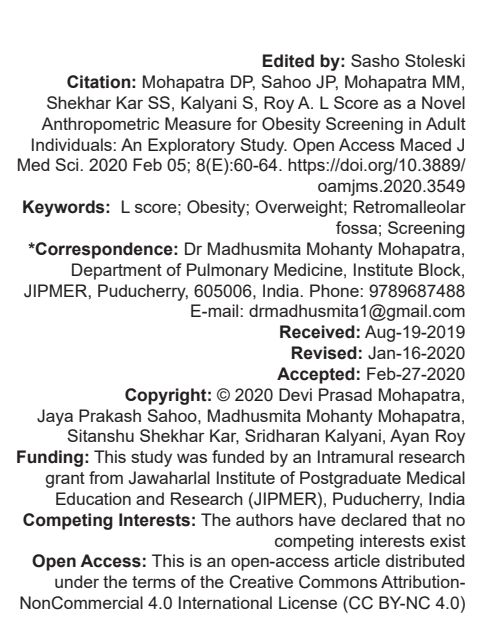

Abstract

BACKGROUND: Obesity is one of today's most neglected public health problems, affecting every region of the world. Early identification of increased weight gain among the population is paramount to prevent the attendant complications associated with obesity.

OBJECTIVES: The primary objective of this study was to measure the distribution of $L$ score in the representative population and the secondary objective was to identify an association between $L$ score values and other measures of obesity such as body mass index, waist circumference, waist-to-height ratio, neck circumference (NC), and tota body fat percentage.

METHODS: This study was conducted in the departments of plastic surgery and endocrinology of a tertiary care institute. The $\mathrm{L}$ score (a measure of fullness of the lateral retromalleolar fossa in the lower limb) was assessed in all the participating individuals. Statistical analysis was performed using the Statistical Package for the Social Sciences version 19.0. $p<0.05$ was considered as statistically significant in statistical analysis.

RESULTS: Among the 50 participants taken in this study, 24 had L score 0,15 had score 1 , and 11 had score 2 . The participants with $\mathrm{L}$ score 1 and 2 had higher obesity, higher $\mathrm{NC}$, and more body fat percentage compared to those having score 0 . All the participants with $\mathrm{L}$ score 2 were overweight and had central obesity.

CONCLUSIONS: The L score measure has a potential for simple and rapid screening of at-risk population for overweight and obesity.

\section{Introduction}

Obesity is one of today's most neglected public health problems, affecting every region of the globe [1]. According to the National Family Health Survey-4, there has been an increase in the prevalence of overweight and obesity among the Indian population (where this study was carried out) as well as many developing and developed countries in the world [2]. The prevalence of overweight and obesity in India ranges from 15\% to $31.3 \%$ for females and $14.3 \%$ to $26.6 \%$ in males [2]. Overweight and obesity are associated with numerous health problems such as cardiovascular diseases, diabetes mellitus, osteoarthritis, gallstone diseases, and probably sex hormone-sensitive cancers [3]. Early identification of increased weight gain among the population is paramount to prevent the attendant complications. The existing methods of measuring body adiposity directly are hydrodensitometry, dualenergy X-ray absorptiometry, magnetic resonance imaging, near-infrared intereactance, and total body electrical conductivity [4]. However, these techniques are expensive and impractical to use as screening tools. Therefore, anthropometric parameters are routinely used as surrogate markers of body fat. However, these methods require equipment and training of screening personnel. In addition, carrying out anthropometric measurements are time consuming when screening is required in areas with high population densities. Hence, we felt the need of a simple technique which could rapidly identify increasing body adiposity among the people at risk in a busy outpatient clinic or a general health screening camp.

The lateral retromalleolar fossa (LRMF) or fossa retromalleolaris lateralis is an anatomically defined concave triangular space behind the lateral malleolus of the lower limb in humans. Anatomically, it is bounded anteriorly by the posterior border of fibula and posterior margin of the lateral malleolus. Posterior border of the space is formed by lateral border of lower third of Achilles tendon. The anterior and posterior border meets superiorly to form the superior angle of the LRMF. The inferior border is formed by an imaginary line drawn from the inferior border of lateral malleolus to tendoachilles 
parallel to the sole of foot. We observed the fullness and obliteration of LRMF in individuals visiting our clinics for consultation of overweight and obesity. We defined the degree of fullness as a score ( $L$ score). As there have been no studies so far on fullness of the LRMF as an indicator for increased body adipose mass, we carried out this study with an aim to find an association between L score, overweight, and obesity.

\section{Materials and Methods}

This cross-sectional study was conducted in plastic surgery and endocrinology departments of a tertiary care institute from July 2017 to June 2018. The study protocol was approved by the Institute Ethics Committee (JIP/IEC/2015/19/704) and written informed consent was obtained from all participants before enrolment in the study. The study was conducted based on the principles outlined in the Declaration of Helsinki. Patients aged between 18 and 60 years attending the outpatient clinic were included in the study. Individuals with lower limb injuries, immobile, bed ridden patients, individuals who had undergone lower limb surgery, as well as those with musculoskeletal deformities and lymphedema involving the lower limb were excluded from the study. The primary objective was to measure the distribution of $\mathrm{L}$ score in the representative population and the secondary objective was to identify an association between $\mathrm{L}$ score values and other measures of obesity such as body mass index (BMI), waist circumference (WC), waist-to-height ratio (WHtR), neck circumference (NC), and total body fat percentage.

The basic demographic characteristics were collected for all subjects using the WHO STEPS questionnaire [5]. All patients underwent a detailed clinical evaluation. Height in centimeter was measured using a stadiometer (SECA Model 214, seca $\mathrm{GmbH}$ \& Co. KG, Hamburg, Germany). Body weight was measured using the electronic scale to the nearest 0.1 $\mathrm{kg}$. BMI was calculated using the standard formula of weight $(\mathrm{kg}) /$ height $^{2}\left(\mathrm{~m}^{2}\right)$. BMl $>23 \mathrm{~kg} / \mathrm{m}^{2}$ and $\mathrm{BMI}>25$ were defined as overweight and obesity, respectively [6]. WC was measured at the midpoint of the inferior costal margin and the superior border of the iliac crest on the mid-axillary line level at the end of expiration using a flexible plastic tape [7]. The healthy WC limits were taken as $90 \mathrm{~cm}$ for men and $80 \mathrm{~cm}$ for women. WHtR was calculated as the WC divided by the height, both measured in centimeter and WHtR $<0.5$ was considered as normal [8]. NC was measured in the midway of the neck, between mid-cervical spine, and mid-anterior neck with both shoulders relaxed [8]. Four sites (biceps, triceps, subscapular, and suprailiac) and skinfold thickness (SFT) were taken in a standardized manner using Harpenden calipers and Durnin and
Womersley formula was used for calculation of total body fat percentage [9]. The average of biceps and triceps SFT was considered as peripheral SFT. The average of subscapular and triceps SFT was considered as truncal SFT. The overall SFT was calculated as the mean of all four SFTs. The arterial blood pressure measurement was done according to the Joint National Committee VII recommendations [10].

A direct observation of LRMF was made with the participant standing barefoot on a flat surface facing away from the examiner. The fullness was scored after comparing with the reference scoring photograph, as shown in Figure 1.

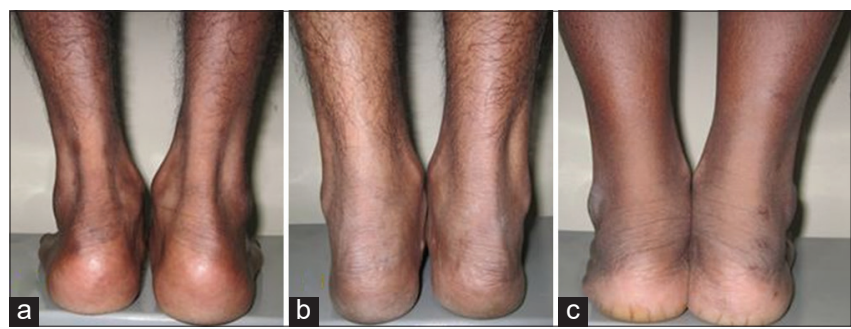

Figure 1: The photograph of lateral retromalleolar fossa showing $L$ score values (a: $L$ Score 0, b: $L$ Score 1, and c: $L$ Score 2)

$\mathrm{L}$ score assessment in this study was done by the principal investigator (DM) and coinvestigator (JPS). The confusion in $L$ score when present was resolved after discussions with other coinvestigators (SK and MMM). No fullness of LRMF was defined as $L$ score 0 , partial fullness of the fossa as $L$ score 1 , and complete fullness and obliteration of the fossa was defined as $L$ score 2 . Before starting this study, an inter-rater variability measurement was carried out. Four independent observers scored 10 individuals using the L score. Inter-rater agreement as measured using Fleiss kappa was $73 \%$ with a kappa score 0.60 which indicated good agreement.

The plasma glucose, lipid profile, and thyroid function tests were measured in the fasting blood sample among the participating individuals. The plasma glucose was measured by glucose oxidase method. Estimation of total cholesterol, high-density lipoprotein (HDL) cholesterol, and triglyceride (TG) was carried out by the colorimetric enzymatic method. The very lowdensity lipoprotein (VLDL) cholesterol was calculated by dividing TG by 5 . The low-density lipoprotein (LDL) cholesterol was calculated from Friedewald's formula (total cholesterol-[HDL + VLDL]) [11]. Serum thyroidstimulating hormone (TSH) and free thyroxine (FT4) were measured by immunoassay using ADVIA Centaur XP, Siemens Healthcare Global, USA. The reference values for the normal TSH and FT4 in our laboratory are $0.35-5.5 \mathrm{mIU} / \mathrm{l}$ and $0.89-1.76 \mathrm{ng} / \mathrm{dl}$, respectively.

Statistical analysis was performed using the Statistical Package for the Social Sciences version 19.0. Kolmogorov-Smirnov test was used to verify data distribution. Continuous variables with and without a normal distribution were expressed as mean \pm standard deviation and median (interquartile range), respectively. Categorical variables were presented as 
the percentage. Unpaired Student's t-test and ANOVA were used to analyse the differences in continuous variables with normal distribution between subjects with different $L$ scores. The Mann-Whitney U-test and Kruskal-Wallis test were used for continuous variables, which were not normally distributed. Chi-square test and Fisher's exact test were used for categorical variables. $p<0.05$ was considered as statistically significant in statistical analysis.

\section{Results}

A total of 50 subjects were included in this crosssectional study. The mean age of the participants was 41 years (range, 18-67). Nearly $50 \%$ of subjects were younger than 40 years. Thirty-one $(62 \%)$ subjects were female, majority of them worked at home. Twenty-eight (56\%) participants were from urban area. Majority of our participants belonged to low socioeconomic status, received a high school education in government schools, and were taking non-vegetarian diet. Around $16 \%$ of the participants had a family history of diabetes and/or hypertension.

The comparison of the study parameters between participants with different $L$ scores is shown in Table 1.

Individuals with $\mathrm{L}$ score 1 and 2 had higher obesity (both generalized and central), thicker SFT, higher NC, more body fat percentage, and higher blood pressure compared to those having score 0 . However, there was no difference in these parameters between the subjects with $L$ score 1 and 2 . We did not notice any difference in fasting plasma glucose, lipid parameters, and thyroid function test between subjects with different $\mathrm{L}$ scores (data not shown).
The prevalence of overweight and obesity among subjects with different $L$ scores is shown in Table 2 and Figure 2.

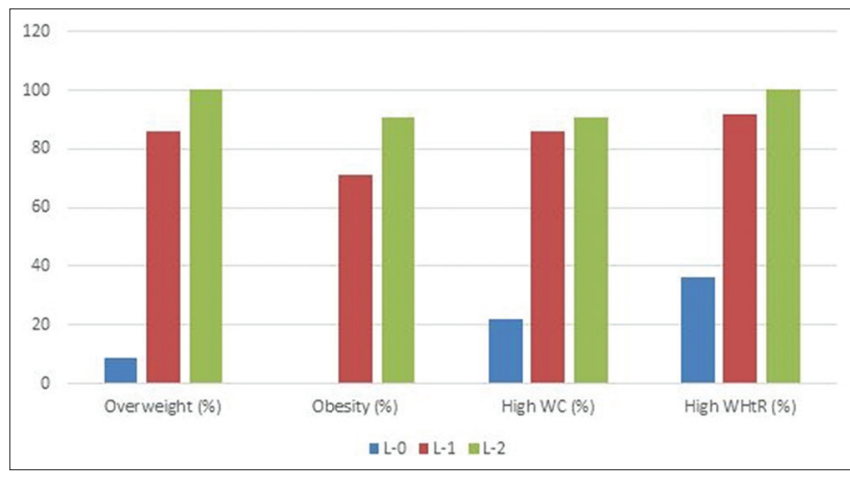

Figure 2: The prevalence of overweight and obesity among subjects with different $L$ scores

Out of total 25 overweight individuals, 20 were obese. Twenty-seven had had central obesity as defined by high WC and 31 participants had central obesity as defined by high WHtR. Among individuals with $L$ score 2, all were overweight and all of them had central obesity as defined by high WHtR while none was obese among participants with $\mathrm{L}$ score 0 (22\% had high W.C and $36 \%$ had central obesity). Individuals with either $L$ score 1 or 2 had higher prevalence of both generalized and central obesity compared to those with $L$ score 0 . However, we noticed that there was no difference in the prevalence of obesity between subjects with $L$ score 1 and L score 2.

\section{Discussion}

Almost all countries including the developing ones, with different cultures and geographic regions

Table 1: Comparison of the study parameters between subjects with different $L$ score values

\begin{tabular}{|c|c|c|c|c|c|c|c|}
\hline Parameters & Score $0(n=24)$ & Score $1(n=15)$ & Score $2(n=11)$ & p-value (0 vs. 1 vs. 2 ) & p-value (0 vs. 1 ) & p-value (0 vs. 2 ) & p-value (1 vs. 2 ) \\
\hline Weight (kg) & $45.6 \pm 7.4$ & $64.3 \pm 14.2$ & $72.5 \pm 8$ & 0.001 & 0.001 & 0.001 & 0.135 \\
\hline${ }^{*} \mathrm{BMl}\left(\mathrm{kg} / \mathrm{m}^{2}\right)$ & $18.9 \pm 2.6$ & $26.7 \pm 3.6$ & $29.6 \pm 5.58$ & 0.001 & 0.001 & 0.001 & 0.19 \\
\hline${ }^{*} \mathrm{SBP}(\mathrm{mmHg})$ & $113 \pm 11$ & $127 \pm 14$ & $128 \pm 13$ & 0.01 & 0.037 & 0.024 & 1.0 \\
\hline${ }^{*} \mathrm{DBP}(\mathrm{mmHg})$ & $71 \pm 10$ & $83 \pm 8$ & $82 \pm 10$ & 0.009 & 0.029 & 0.030 & 1.0 \\
\hline Waist/height ratio & $0.49 \pm 0.5$ & $0.59 \pm 0.07$ & $0.63 \pm 0.08$ & 0.001 & 0.001 & 0.001 & 0.251 \\
\hline $\mathrm{NC}(\mathrm{cm})$ & $32.3 \pm 4$ & $36.6 \pm 6.5$ & $36.6 \pm 2.2$ & 0.008 & 0.023 & 0.04 & 1.0 \\
\hline Neck/height ratio & $0.21 \pm 0.03$ & $0.23 \pm 0.23$ & $0.23 \pm 0.01$ & 0.005 & 0.016 & 0.027 & 1.0 \\
\hline Triceps * ${ }^{*} \mathrm{SFT}(\mathrm{mm})$ & $8.6 \pm 4.8$ & $20.7 \pm 21$ & $22.9 \pm 9.9$ & 0.001 & 0.001 & 0.001 & 1.0 \\
\hline Biceps *SFT (mm) & $4.2(2.8-5.6)$ & $13(4.8-21)$ & $14(8.2-25)$ & 0.001 & 0.003 & 0.001 & 0.377 \\
\hline Subscapular *SFT (mm) & $9(5.4-19)$ & $28(19-35)$ & $26(20-32)$ & 0.001 & 0.001 & 0.001 & 0.775 \\
\hline Suprailiac *SFT $(\mathrm{mm})$ & $12.3 \pm 8.4$ & $27.5 \pm 31$ & $34.8 \pm 9$ & 0.001 & 0.001 & 0.001 & 0.225 \\
\hline Truncal *SFT $(\mathrm{mm})$ & $11.9 \pm 7.5$ & $26.9 \pm 12.8$ & $30.9 \pm 7.3$ & 0.001 & 0.001 & 0.001 & 1.0 \\
\hline Average *SFT $(\mathrm{mm})$ & $9.4 \pm 8.5$ & $22 \pm 10.4$ & $24.9 \pm 7.3$ & 0.001 & 0.001 & 0.001 & 1.0 \\
\hline Percentage body fat (\%) & $19.8(12.2-28.2)$ & $38.7(30-41.8)$ & $38.3(29.3-43.1)$ & 0.001 & 0.002 & 0.001 & 0.856 \\
\hline
\end{tabular}

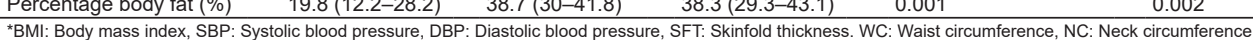

Table 2: Prevalence of overweight and obesity among subjects with different $L$ score values

\begin{tabular}{|c|c|c|c|c|c|c|c|}
\hline Parameters & Score $0(\%)$ & Score $1(\%)$ & Score $2(\%)$ & p-value (0 vs. 1 vs. 2 ) & p-value (0 vs. 1 ) & p-value (0 vs. 2 ) & p-value (1 vs. 2) \\
\hline Overweight ( ${ }^{*} \mathrm{BMI} \geq 23 \mathrm{~kg} / \mathrm{m}^{2}$ ) & $2 / 23(9)$ & $12 / 14(86)$ & $11 / 11(100)$ & $<0.001$ & $<0.001$ & $<0.001$ & 0.30 \\
\hline Obesity $\left({ }^{*} \mathrm{BMI} \geq 25 \mathrm{~kg} / \mathrm{m}^{2}\right)$ & $0 / 23$ & 10/14 (71) & 10/11 (91) & $<0.001$ & $<0.001$ & $<0.001$ & 0.28 \\
\hline Central obesity (high *WC) & $5 / 23(22)$ & $12 / 14(86)$ & 10/11 (91) & $<0.001$ & $<0.001$ & $<0.001$ & 0.75 \\
\hline Central obesity (*WHtR $\geq 0.5$ ) & $8 / 22(36)$ & $12 / 13(92)$ & $11 / 11(100)$ & $<0.001$ & $<0.001$ & $<0.001$ & 0.76 \\
\hline
\end{tabular}


are facing an escalating trend of obesity. While early detection is a key to prevention of this global pandemic, no existing techniques for identifying overweight and obesity are convenient enough to be implemented in large cross sections of population in a limited time. This constraint becomes more pronounced in developing countries with huge population density. The inability to detect overweight as well as obesity early would lead to millions of people suffering from the consequences of the disorders associated with these conditions. A simple technique like $\mathrm{L}$ score measurement has a potential to simplify screening of at risk population for overweight and obesity while attending a busy clinic in a public hospital or general health camps.

Increasing obesity in Asians in general and Indians particularly is primarily driven by stress, demographic transitions, unhealthy diets, and physical inactivity, in the background of genetic predisposition [12]. Our study showed that $50 \%$ of subjects were overweight and $62 \%$ of participants had central obesity which may be explained by the fact that majority of our participants were female (62\%) and belonged to urban population, which are known as risk factors for obesity among South Asians [12]. Central obesity has been found to have good association with metabolic syndrome including cardiovascular diseases and Southeast Asian populations are known to develop abdominal obesity with lesser degree of generalized obesity [6]. BMI and total body fat percentage reflect generalized obesity, while WC and WHtR are indicators of central obesity [7], [13]. In addition, NC is associated with obstructive sleep apnea, which is a risk factor for sudden cardiac death in obese persons [14].

Although there was no difference in adiposity between subjects with L1 and L2 scores in our study, they had higher prevalence of both central and generalized obesity compared to those with L0 score. In addition, none with $L$ score 0 was found to be obese while all subjects with $L$ score 2 had overweight and central obesity. This means that subjects with $L$ score 0 do not need further evaluation to rule out overweight or obesity and associated complications. This would reduce unnecessary work burden on health care workers without compromising the quality of health care with efficient utilization of available limited medical resources, especially in developing countries.

Anthropometric measures need removal of clothing which may be inconvenient or embarrassing to individuals while $\mathrm{L}$ score measurement relies, only on observing the surface anatomy of the retromalleolar region in the lower leg without the need to remove garments. Specialized health workers are not needed for this purpose and this parameter can even be conveniently measured by the individuals themselves. The $L$ score even can provide a guide for changing body fat over a period of time. Individuals can assess their $L$ score over a period of time, for example, monthly intervals and turn up at a specialized clinic for further measurements if they notice an increasing $L$ score. Although the participants in this study are less in number, the major strength of our study is that we have devised and used a novel anthropometric parameter for rapid screening of obesity.

\section{Conclusions}

The Lscore is a novel and simple anthropometric measure designed to evaluate the distribution of obesity in a large population within a short period of time. A further larger study including pediatric population as well as different ethnic groups is required to assess the utility of this measure. In addition, the correlation of $L$ scores with metabolic syndrome and changes in $L$ score with time needs to be done in different populations in the future.

\section{Acknowledgment}

The first and second authors have equal contribution in design and execution of this project.

\section{References}

1. NCD Risk Factor Collaboration (NCD-RisC). Worldwide trends in body-mass index, underweight, overweight, and obesity from 1975 to 2016: A pooled analysis of 2416 population-based measurement studies in 128.9 million children, adolescents, and adults. Lancet. 2017;390(10113):2627-42. https://doi. org/10.1530/ey.15.13.20

PMid:29029897

2. India Fact Sheet, National Family Health Survey-4, 2015-16. Available from: http://www.rchiips.org/nfhs/pdf/NFHS4/India. pdf. [Last accessed on 2019 Jan 21].

3. Kinlen D, Cody D, O'Shea D. Complications of obesity. QJM. 2018;111(7):437-43. https://doi.org/10.1093/qjmed/hcx152 PMid:29025162

4. Duren DL, Sherwood RJ, Czerwinski SA, Lee M, Choh AC Siervogel RM, et al. Body composition methods: Comparisons and interpretation. J Diabetes Sci Technol. 2008;2(6):1139-46. https://doi.org/10.1177/193229680800200623

PMid:19885303

5. The WHO STEP Wise Approach to Chronic Disease Risk Factor Surveillance (STEPS) Instrument (Core and Expanded). Available from: http://www.who.int/ncds/surveillance/steps/ STEPS_Instrument_v2.1.pdf. [Last accessed on 2019 Jan 20].

6. Misra A, Chowbey P, Makkar BM, Vikram NK, Wasir JS, Chadha $\mathrm{D}$, et al. Consensus statement for diagnosis of obesity, abdominal obesity and the metabolic syndrome for Asian Indians 
and recommendations for physical activity, medical and surgical management. J Assoc Physicians India. 2009;57:163-70.

PMid:19582986

7. Agarwal SK, Misra A, Aggarwal P, Bardia A, Goel R, Vikram NK, et al. Waist circumference measurement by site, posture, respiratory phase, and meal time: Implications for methodology. Obesity (Silver Spring). 2009;17(5):1056-61. https://doi. org/10.1038/oby.2008.635

PMid:19165166

8. Verma M, Rajput M, Sahoo SS, Kaur N. Neck circumference: Independent predictor for overweight and obesity in adult population. Indian J Community Med. 2017;42(4):209-13. https://doi.org/10.4103/ijcm.ijcm_196_16

PMid:29184320

9. Durnin JV, Womersley J. Body fat assessed from total body density and its estimation from skinfold thickness: Measurements on 481 men and women aged from 16 to 72 years. Br J Nutr. 1974;32(1):77-97. https://doi.org/10.1079/bjn19740060 PMid:4843734

10. Chobanian AV, Bakris GL, Black HR, Cushman WC, Green LA Izzo JL Jr., et al. The seventh report of the joint national committee on prevention, detection, evaluation, and treatment of high blood pressure. JAMA. 2003;289(19):2560-72. https:// doi.org/10.1001/jama.289.19.2560

PMid:12748199

11. Friedewald WT, Levy RI, Fredrickson DS. Estimation of the concentration of low-density lipoprotein cholesterol in plasma, without use of the preparative ultracentrifuge. Clin Chem. 1972;18(6):499-502. https://doi.org/10.1093/clinchem/18.6.499 PMid:4337382

12. Misra A, Shrivastava U. Obesity and dyslipidemia in South Asians. Nutrients. 2013;5(7):2708-33. https://doi.org/10.3390/ nu5072708

PMid:23863826

13. Romero-Saldaña $M$, Tauler $P$, Vaquero-Abellán $M$, LópezGonzález AA, Fuentes-Jiménez FJ, Aguiló A, et al. Validation of a non-invasive method for the early detection of metabolic syndrome: A diagnostic accuracy test in a working population. BMJ Open. 2018;8(10):e020476. https://doi.org/10.1136/ bmjopen-2017-020476

PMid:30344164

14. Ahbab S, Ataoğlu HE, Tuna M, Karasulu L, Cetin F, Temiz LU, et al. Neck circumference, metabolic syndrome and obstructive sleep apnea syndrome; evaluation of possible linkage. Med Sci Monit. 2013;19:111-7. https://doi.org/10.12659/msm.883776 PMid:23403781 